\title{
Inverse proximity effects at spin-triplet superconductor-ferromagnet interface
}

\author{
O. Maistrenko, ${ }^{1}$ C. Autieri $\odot,{ }^{2,3}$ G. Livanas $\odot,{ }^{3,4}$ P. Gentile,,${ }^{3,5}$ A. Romano,,${ }^{5,3}$ C. Noce $\odot,{ }^{5,3}$ D. Manske,,${ }^{1}$ and M. Cuoco $\odot^{3,5}$ \\ ${ }^{1}$ Max Planck Institute for Solid State Research, D-70569 Stuttgart, Germany \\ ${ }^{2}$ International Research Centre MagTop, Institute of Physics, Polish Academy of Sciences, Aleja Lotników 32/46, PL-02668 Warsaw, Poland \\ ${ }^{3}$ SPIN-CNR, c/o Università di Salerno, IT-84084 Fisciano (SA), Italy \\ ${ }^{4}$ Department of Physics, National Technical University of Athens, GR-15780 Athens, Greece \\ ${ }^{5}$ Dipartimento di Fisica “E. R. Caianiello”, Università di Salerno, IT-84084 Fisciano (SA), Italy
}

(Received 24 December 2020; revised 28 April 2021; accepted 18 May 2021; published 2 July 2021)

\begin{abstract}
We investigate proximity effects in a spin-triplet superconductor (TSC) interfaced with a ferromagnet (FM), assuming different types of magnetic profiles and chiral or helical pairings. The region of the coexistence of spin-triplet superconductivity and magnetism is significantly influenced by the orientation and spatial extension of the magnetization with respect to the spin configuration of the Cooper pairs, resulting in clear-cut anisotropy signatures. A characteristic mark of the inverse proximity effect arises in the induced spin polarization at the TSC-FM interface. This is unexpectedly stronger when the magnetic proximity is weaker, thus unveiling immediate detection signatures for spin-triplet pairs. We show that an anomalous magnetic proximity can occur at the interface between the itinerant ferromagnet $\mathrm{SrRuO}_{3}$ and the unconventional superconductor $\mathrm{Sr}_{2} \mathrm{RuO}_{4}$. Such scenario indicates the potential to design characteristic inverse proximity effects in experimentally available $\mathrm{SrRuO}_{3}-\mathrm{Sr}_{2} \mathrm{RuO}_{4}$ heterostructures and to assess the occurrence of spin-triplet pairs in the highly debated superconducting phase of $\mathrm{Sr}_{2} \mathrm{RuO}_{4}$.
\end{abstract}

DOI: 10.1103/PhysRevResearch.3.033008

\section{INTRODUCTION}

Spin-triplet superconductors can be odd parity in momentum, e.g., a $p$ wave [1-8], or even parity for multiorbital configurations [9-13]. Apart from the variety of orbital pairings, the spin-triplet part of the superconducting (SC) order parameter (OP), typically encoded in a $\mathbf{d}$ vector, has a substantial imprint on the superconducting properties. In fact, the spin degree of freedom in superconductors naturally enriches the physical scenario, resulting in an anomalous response to Zeeman or ferromagnetic fields [14-20], spinsensitive Josephson transport [21-29], magnetic topological reconstructions [18-20], and, on a general ground, may lead to energy-efficient superconducting spintronics [30]. Notably, intrinsic or engineered spin-triplet superconductors can host Majorana bound states and thus are particularly impactful for topological quantum computing [4,31-33].

While more rare in nature than the canonical spin-singlet one, several experimental observations have led to evidence for spin-triplet superconductivity in a large variety of materials [34-47]. A paradigmatic case is $\mathrm{Sr}_{2} \mathrm{RuO}_{4}$, whose superconducting nature is under intense debate because recent measurements [48-50] pose serious constraints on the long-thought spin-triplet chiral $p$-wave superconductivity [51]

Published by the American Physical Society under the terms of the Creative Commons Attribution 4.0 International license. Further distribution of this work must maintain attribution to the author(s) and the published article's title, journal citation, and DOI. proposed to characterize that system. Such case underlines the remarkable challenges that are typically encountered in assessing the occurrence of spin-triplet pairing in unconventional superconductors.

In this work, we take an alternative exploratory path to access the spin-triplet nature of the superconducting pairing by focusing on its spin degree of freedom and investigating the inverse proximity effects that can occur at the spin-triplet superconductor-ferromagnet (TSC-FM) interface, assuming that spin polarization may leak into the TSC. Inverse proximity effects have been studied in heterostructures involving spin-singlet superconductors [52-55] with unexpected consequences when nodal excitations occur [56]. Instead, while it is well confirmed that the physics of TSC-FM heterostructures is richer than their singlet counterparts because the orientation of the FM moment relative to the TSC $\mathbf{d}$ vector introduces extra channels of coupling, to the best of our knowledge, the proximity effects both for the magnetic and superconducting components in TSC-FM have not yet been fully addressed. There are various fundamental physical aspects to be accounted for once the spin polarization of the FM penetrates into the TSC regarding the reconstruction of the SC-OP and the modification of the magnetic proximity (MP) due to the presence of the spin-triplet pairs. The analysis is performed by considering two prototypical spin-triplet pairings with uniaxial and planar $\mathbf{d}$-vector and various characteristic spatial profiles and orientations of the magnetization [Figs. 1(a)1(d)] in the TSC with inequivalent character of the magnetic proximity [Fig. 1(e)]. The investigation focuses on the case of layered superconductors whose out-of-plane superconducting coherence length $\left(\xi_{S}\right)$ is of the order of several unit cells along 
(a)

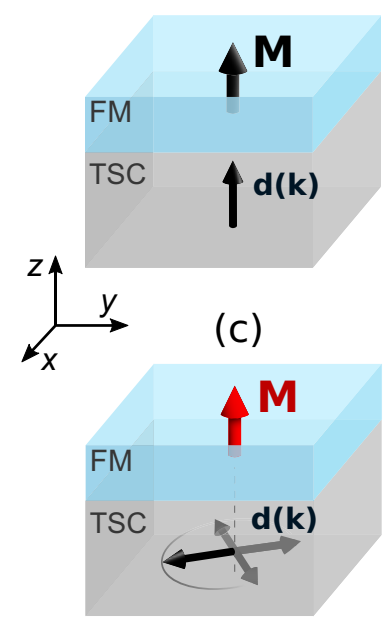

(b)

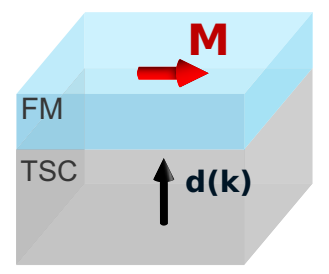

(d)

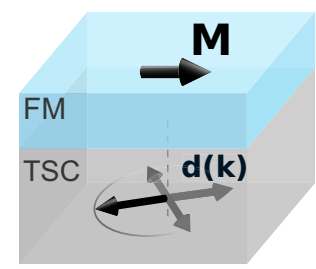

(e)

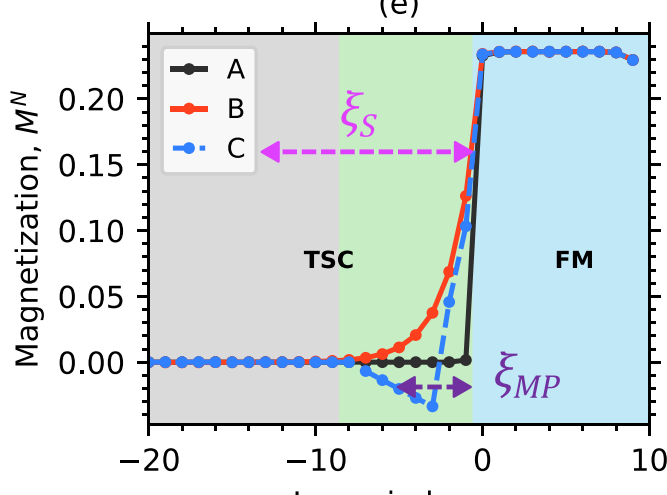

Layer index, $z$
FIG. 1. Illustration of the magnetic and superconducting configurations for the TSC-FM heterostructure with (a), (b) chiral and (c), (d) helical order parameters, respectively. The magnetization is (a), (c) out-of-plane or (b), (d) in-plane oriented. (e) The examined magnetic profiles can have (A) a steplike magnetization at the interface with substantial absence of magnetic proximity, (B) a monotonous decaying of the spin polarization within the TSC on the scale of the superconducting coherence length, or $(\mathrm{C})$ a sign-changing penetration in the TSC, respectively. The blue region stands for the ferromagnetic layers. In the gray area, the spin polarization induced by the FM into the TSC has a vanishing amplitude. $\xi_{S}$ and $\xi_{\mathrm{MP}}$ are the superconducting coherence length and the magnetic proximity distance, respectively. The magnetic proximity length refers to the penetration of the spin polarization in the normal metal-ferromagnet configuration.

the direction perpendicular to the interface and the magnetic leaking distance $\left(\xi_{\mathrm{MP}}\right)$ is smaller than $\xi_{S}$, being of the order of few unit cells [Fig. 1(e)]. We find that the TSC order parameter in the region of the magnetic-superconducting coexistence is significantly sensitive to the orientation of the magnetization, thus unveiling clear-cut anisotropy signatures. Similarly, the behavior of the induced spin polarization in the TSC depends on the character of the $\mathbf{d}$ vector and on the inverse magnetic proximity pattern. Then, we consider heterostructures that are expected to be more suitable for achieving the investigated magnetic proximity profiles and be employed to design a superconductor-ferromagnet interface with a high degree of electronic matching at the interface. In this context, oxide interfaces are ideal because they are atomically controlled and one can reduce the disorder at the interface. Moreover, the heterostructure made of the itinerant ferromagnet $\mathrm{SrRuO}_{3}$ and the unconventional superconductor $\mathrm{Sr}_{2} \mathrm{RuO}_{4}$ represents a notable configuration since the electronic structures of the ferromagnet and of the superconductor arise from $d$ states of the same transition metal element. The choice of the $\mathrm{Sr}_{2} \mathrm{RuO}_{4}-\mathrm{SrRuO}_{3}$ heterostructure is also motivated by the timely debate on the nature of the superconducting phase in $\mathrm{Sr}_{2} \mathrm{RuO}_{4}[48,50]$ and on its anomalous magnetotransport properties [57-59]. Along this line, we evaluate the MP of the $\mathrm{Sr}_{2} \mathrm{RuO}_{4}$ when interfaced with $\mathrm{SrRuO}_{3}$ by means of density functional theory methods. We find that the $\mathrm{SrRuO}_{3}$ induces a large magnetic moment in the $\mathrm{Sr}_{2} \mathrm{RuO}_{4}$ interface layers with characteristic profiles that depend on the strength of the Coulomb interaction and strain at the interface. This magnetic scenario supports the general investigation of the inverse proximity effects in the presence of different types of magnetic leakage into the TSC. The recent developments in the fabrication of $\mathrm{Sr}_{2} \mathrm{RuO}_{4}$ thin films [60-63] and heterostructures allow one to experimentally investigate the properties of the inverse proximity effect, which we discuss in our work. This can provide relevant experimental paths to address the debate about the nature of the superconductivity in $\mathrm{Sr}_{2} \mathrm{RuO}_{4}$.

The paper is organized as follows. In Sec. II, we present the model and the methodology for the evaluation of the proximity effects. Section III is devoted to the main results by focusing on the evolution of the superconducting order parameter and the spin polarization at the interface. In Sec. III, we present the magnetic proximity for a realistic normal metal-ferromagnet heterostructure by considering the $\mathrm{Sr}_{2} \mathrm{RuO}_{4}-\mathrm{SrRuO}_{3}$ interface. In Sec. IV, we provide the discussion on the resulting effects and the concluding remarks. Finally, in the Appendices, we compare an effective monolayer and bilayer geometry with spin-triplet superconductivity and present the details of the density functional theory computation employed for the $\mathrm{Sr}_{2} \mathrm{RuO}_{4}-\mathrm{SrRuO}_{3}$ heterostructure.

\section{MODEL AND METHODOLOGY}

We consider a FM-TSC heterostructure with a layered geometry described by a single-band tight-binding model with a spin-dependent nearest-neighbor attractive interaction that can stabilize spin-triplet pairing with chiral or helical d vectors (Fig. 1) $[24,25,64,65]$. The total Hamiltonian of the system is then defined on a lattice with size $L_{x} \times L_{y} \times L_{z}$ ( $L_{x}=L_{y}=L$ ) assuming periodic boundary conditions along $x$ and $y$. The simulation is performed for a system having $L=100$ and $L_{z}=40$. Since the out-of-plane superconducting coherence length is of the order of about six unit cells, the size of the system is adequate for tracking the inverse and direct proximity effects on the characteristic superconducting length scales. We indicate each site by a vector $\vec{i} \equiv\left(i, i_{z}\right)$, with $i$ labeling the $(x y)$ in-plane atomic positions and $i_{z}$ the different layers along the $z$ direction. The FM region corresponds to layers with $i_{z} \geqslant 0$ and the TSC one to layers with $i_{z}<0$, 
respectively. The Hamiltonian can be expressed as

$$
\begin{aligned}
H= & \sum_{\mathbf{k}, i_{z}, \sigma}\left(\epsilon_{\mathbf{k} \sigma}-\mu\right) c_{\mathbf{k} \sigma}^{\dagger}\left(i_{z}\right) c_{\mathbf{k} \sigma}\left(i_{z}\right)-\sum_{i_{z}} \mathbf{h}\left(i_{z}\right) \cdot \mathbf{M}\left(i_{z}\right) \\
& -\sum_{\langle i j\rangle, i_{z}<0, \sigma, \sigma^{\prime}} V_{\sigma, \sigma^{\prime}} n_{i \sigma}\left(i_{z}\right) n_{j \sigma^{\prime}}\left(i_{z}\right) \\
& +\sum_{\delta= \pm 1} \sum_{\mathbf{k} \sigma, i_{z}} t_{\perp}\left[c_{\mathbf{k} \sigma}^{\dagger}\left(i_{z}+\delta\right) c_{\mathbf{k} \sigma}\left(i_{z}\right)+\text { H.c. }\right],
\end{aligned}
$$

with $c_{\mathbf{k} \sigma}\left(i_{z}\right)$ being the annihilation operator of an electron with planar momentum $\mathbf{k}$, spin $\sigma$ at the layer $i_{z}$, and $\langle i j\rangle$ denotes the in-plane nearest-neighbor sites, $\mu$ is the chemical potential, $V_{\sigma, \sigma^{\prime}}$ is the spin-dependent in-plane pairing strength, $\mathbf{M}\left(i_{z}\right)=\sum_{i, s, s^{\prime}} c_{s}^{\dagger}(i) \boldsymbol{\sigma}_{s, s^{\prime}} c_{s^{\prime}}(i)$ is the spin density of the layer $i_{z}$, and $\epsilon_{k \sigma}=-2 t\left[\cos \left(k_{x}\right)+\cos \left(k_{y}\right)\right]$ is the in-plane electronic spectrum. $\mathbf{h}\left(i_{z}\right)$ is the layer-dependent exchange field that sets the amplitude and orientation of the magnetization within the heterostructure (Fig. 1). We assume a good electronic matching at the interface expressed by having $t_{\perp}=0.5 t$. The selection of the three profiles in Fig. 1 is general enough to include the phenomenology which is expected when interfacing a correlated paramagnetic metal with a ferromagnet. In fact, the breaking of the translational symmetry due to the interface allows one to get a reconstruction of the MP with monotonous or oscillating spatial behavior. Such occurrence is also expected to be most likely to occur in paramagnetic electronic systems having a peak at finite momentum in the magnetic susceptibility. Furthermore, since the Fermi length is of the order of the unit cell dimension, the MP is taken as more significant in the layers near the interface.

For the present study, the quartic term of the model Hamiltonian in Eq. (1) is decoupled in the canonical HartreeFock approximation within the pairing channel. We introduce the layer-dependent superconducting pairing amplitude for each spin-triplet channel to self-consistently determine the corresponding order parameters with chiral and helical symmetry as

$$
\begin{aligned}
F_{\theta}^{0} & =\frac{1}{L^{2}} \sum_{\sigma \neq \sigma^{\prime}, \mathbf{k}}\left(\sin k_{x}-i \theta \sin k_{y}\right)\left\langle c_{-\mathbf{k} \sigma} c_{\mathbf{k} \sigma^{\prime}}\right\rangle, \\
F_{\theta}^{\sigma} & =\frac{1}{L^{2}} \sum_{\mathbf{k}}\left(\sin k_{x}-i \theta \sin k_{y}\right)\left\langle c_{-\mathbf{k} \sigma} c_{\mathbf{k} \sigma}\right\rangle,
\end{aligned}
$$

where $\theta= \pm$ is employed to set the winding of the spindependent order parameter, while $\langle\cdot\rangle$ is the average over the ground state. Depending on the amplitudes of $V_{\sigma, \sigma^{\prime}}$, the chiral or helical spin-triplet superconductivity can be stabilized $[24,27,65]$.

\section{RESULTS}

In this section, we will present the results concerning the spatial modification of the order parameter across the interface for both chiral and helical spin-triplet pairing, assuming that the magnetization can be collinear or perpendicularly oriented with respect to the $\mathbf{d}$ vector and spatially extended into the superconductor with different character of the magnetic profile.

\section{A. Inverse superconducting and magnetic proximity effects}

The magnetic and superconducting proximity effects on both sides of the interface are expected to exhibit a qualitative behavior which is substantially dictated by two main physical aspects. First, the relative orientation of the $\mathbf{d}$ vector with respect to the magnetic orientation plays a role in setting out the strength of pair breaking effects and thus it directly influences the induced spin polarization and the spatial profile (monotonous or oscillating) of the superconducting order parameter. The second relevant element is related to the interplay between the gradient of the superconducting order parameter and the spin polarization in the region of coexistence close to the interface. The coupling of superconducting and magnetic gradients at the interface can result in a variety of spin-polarization reconstructions. Since the magnetization in the normal-ferromagnet configuration can change its profile within a few unit cells, the qualitative behavior can be grasped by taking an effective model of two coupled layers with inequivalent amplitude of magnetization and of $\mathbf{d}$ vectors (see the Appendices for details). The outcome of the induced spin polarization is expected to be strikingly different from the uniform configuration since there are no a priori constraints on its sign and amplitude variations.

Let us start by discussing the superconducting proximity effects for the chiral $d_{z}$-TSC (Fig. 2), assuming three different magnetization leakages into the TSC [Fig. 1(e)]. The chiral amplitude $F_{+}^{0}$ depends on the orientation of the FM spin polarization [Fig. 2(a)], exhibiting a suppression of its value when the magnetization is parallel $\left(M_{z}\right)$ to the $\mathbf{d}$ vector compared to the transverse configuration $\left(M_{x}\right)$, as schematically shown in Figs. 1(a) and 1(b).

Here, the use of different MP profiles allows us to assess how the leakage of spin polarization in the TSC affects the SC-OP and the resulting spin polarizability of the TSC at the interface.

First, we observe that in the magnetized region of the TSC, the amplitude of the chiral order parameters decreases for the $M_{z}$ orientation, while it is substantially unaffected for the transverse $M_{x}$ case. This is consistent with the spin orientation of the Cooper pairs. The behavior with transverse spin-polarization $M_{x}$-FM [Figs. 2(b) and 2(c)] unveils a weak enhancement when the inverse MP increases in length penetration. Such outcome substantially indicates that the spin polarization entering into the TSC yields more spin-triplet pairs for the proximity into the FM. For the $M_{z}$ state, we notice that the expected damped oscillation of the pairing amplitude does not exhibit variation as a function of the MP.

Let us now focus on the behavior of the induced spin polarization due to the leakage or lack of magnetization within the TSC region of the heterostructure. From the behavior of the $\mathrm{SC}$ order parameter, it is immediate to observe that the effects in the TSC are tied to the orientation of the magnetization with respect to the $\mathbf{d}$ vector. Then, in order to relate the behavior of the induced magnetization to the character of the MP, the strategy is to compute the magnetization difference for each layer of the superconducting state with respect to that of the normal metal configuration, i.e., the one corresponding to a vanishing pairing interaction $V$. This physical quantity gives an indication of the response of the superconductor close to 



FIG. 2. Evolution of the pairing amplitude for chiral TSC heterostructure near the TSC-FM interface assuming different magnetization profiles as reported in Fig. 1(e). (a)-(c) Steplike, monotonous decay, and sign-changing spatial dependence of the magnetization, respectively. The black circles (red squares) refer to a configuration with magnetization oriented along the out-of-plane $z$ direction (in-plane $x$ direction). The $z$ - ( $x$-)oriented spin polarization is collinear (transverse) to the d vector. (d)-(f) The spatial dependence of the difference, $\Delta M$, between the magnetization in the superconducting $\left(M^{S C}\right)$ and normal state $\left(M^{N}\right)$ for the parallel (circles) and transverse (squares) configurations, respectively. The dashed line in (d)-(f) indicates the amplitude of the magnetization in the normal metal state. The green area indicates the layers where the magnetization penetrates into the TSC and there is a coexistence of nonvanishing magnetization and superconductivity. The blue region stands for the ferromagnetic layers. In the gray area, the spin polarization induced by the FM is substantially zero.

the interface, not only for the presence of a nonvanishing exchange field in the region where the order parameter is typically reduced in amplitude, but also to the gradient of the spin polarization which is particularly enhanced at the TSC-FM boundary. In Figs. 2(d)-2(f), we follow the spatial evolution of the induced magnetization for the various MP profiles. As a general trend, we observe that the magnetization change between the superconducting and the normal states is substantially negligible for the $M_{x}$ orientation, i.e., transverse to the d vector with the exception of the layers very close to the interface. There, the induced magnetization typically changes sign irrespective of the MP profile, thus indicating that the TSC tends to screen the MP by spatially modulating the spin polarization. For the transverse magnetization, it is interesting to link the enhancement of the SC proximity effect [Fig. 2(c)] with that of the spin polarization in Fig. 2(f). The result indicates that an increase of the magnetization can be observed at a distance of several layers from the interface due to the penetration of the spin-triplet Cooper pairs with spin polarization collinear to $M$. On the other hand, the response of the TSC to the $M_{z}$ leakage, i.e., parallel to the $\mathbf{d}$ vector, is typically larger in amplitude and significantly sensitive to the character of the MP. We find that for the case of a steplike magnetization at the interface, the response of the TSC is paramagnetic [Fig. 2(d), i.e., the spin polarization is enhanced with respect to the normal state], with a penetration in the superconductor over a spatial range of the order of the out-ofplane coherence length. The impact of the MP is significantly different when considering the case of a longer-range penetration of the magnetization in the TSC with monotonous decay
[Fig. 2(e)]. In this regime, the amplitude of the induced spin polarization spatially oscillates around the one in the normalstate configuration. Thus, the TSC yields a vanishing net spin polarization, on average. When considering the sign-changing MP [Fig. 2(f)], we find that the magnetic reconstruction due to the TSC leads to an overall paramagnetic response with an enhancement of the spin polarization distribution in all layers close to the interface. We argue that the variation of the induced magnetic effects is mainly due to the gradient of the magnetization close to the interface because the amplitude is comparable for the two configurations. In fact, the character of the observed induced spin polarization near the TSC-FM interface can be grasped by analyzing a simplified effective model with only two superconducting layers in the presence of an inhomogeneous exchange field. The details of the outcome of such effective model are reported in Appendices. The changeover from paramagnetic to diamagnetic of the TSC response as a function of the layer position can be ascribed to the gradient of the exchange field rather than to the effective strength of the magnetization with respect to the SC gap (see the Appendices).

At this stage, it is worth asking whether and how the magnetic reconstruction at the TSC-FM gets modified if one is considering a TSC with helical pairing, i.e., $F^{0}=0$ and $F_{+}^{\uparrow}=F_{-}^{\downarrow}$ thus marked by a two-component $\mathbf{d}$ vector lying in the $x y$ plane, as shown in Figs. 1(c) and 1(d). For the helical TSC, the superconducting proximity does not exhibit an oscillating behavior because the $\mathbf{d}$ vector lies in a plane and thus one cannot find a magnetization direction that is fully collinear to it across the whole Brillouin zone. The 

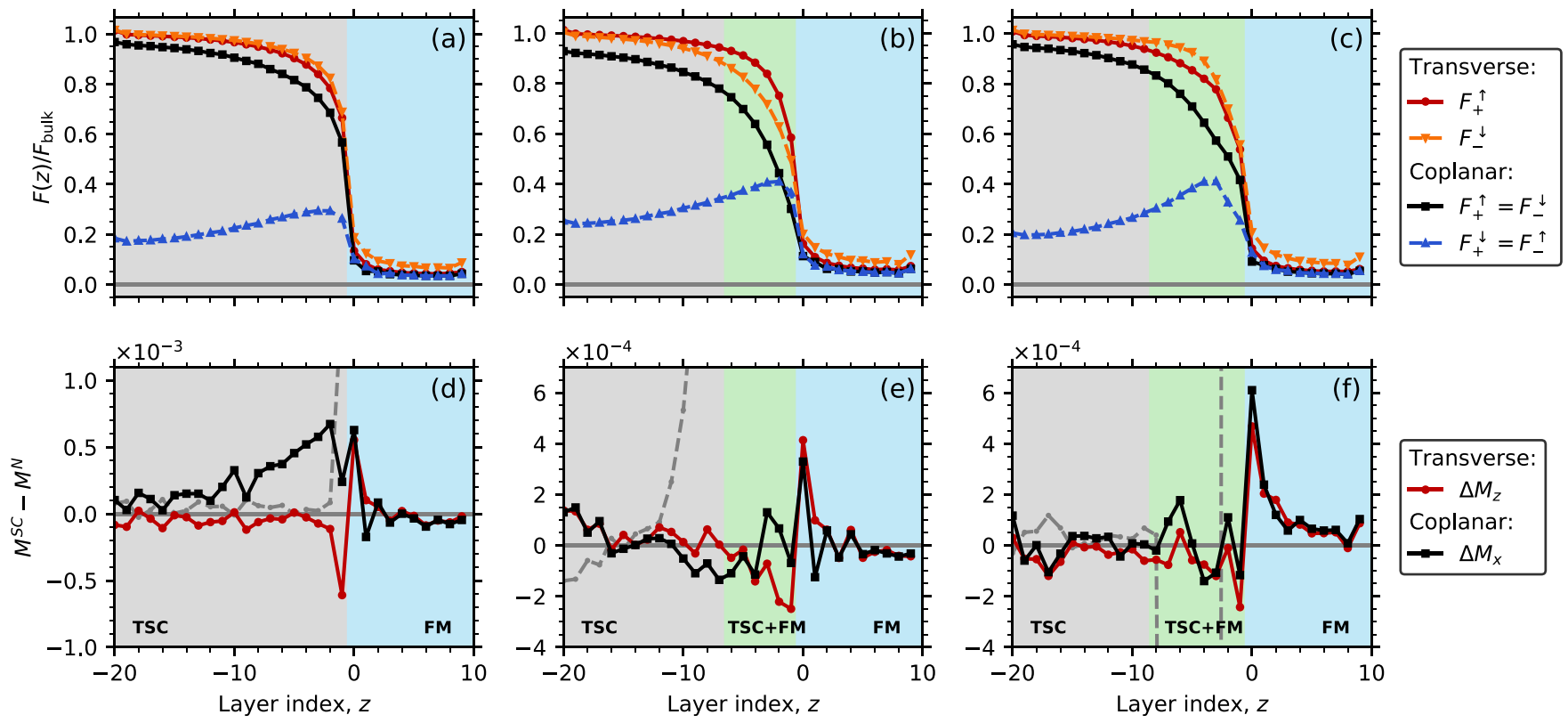

FIG. 3. Evolution of the pairing amplitude for the helical-TSC heterostructure near the TSC-FM interface, assuming different magnetization profiles in the TSC, as reported in Fig. 1(e). (a)-(c) Steplike, monotonous decay, and sign-changing spatial dependence of the magnetization, respectively. Circles (squares) stand for a magnetization which is oriented along the out-of-plane $z$ direction (in-plane $x$ direction). The $z$ - $(x$-)oriented spin polarization is transverse (coplanar) to the d vector. (d)-(f) The spatial dependence of the difference, $\Delta M$, between the magnetization in the superconducting $\left(M^{S C}\right)$ and in the normal $\left(M^{N}\right)$ state with coplanar and transverse configurations. The dashed gray line in (d)-(f) indicates the amplitude of the magnetization in the normal metal for the different types of examined magnetic proximity.

behavior of the TSC pairing amplitude is not much affected by the change of the MP [Figs. 3(a)-3(c)]. Moreover, for the coplanar configuration, the presence of the magnetic interface induces a pairing amplitude in each spin channel with opposite winding compared to that of the SC order parameter, and this is irrespective of the character of the MP. Such component can yield a transverse contribution in the spin polarization with respect to the magnetization orientation. More interesting is the behavior of the spin polarization in the TSC. One finds that for the steplike magnetization profile, the response of the TSC is paramagneticlike for the coplanar configuration, while it is diamagneticlike for the transverse $M_{z}$ state [see Fig. 3(d)]. The effect typically extends over a distance of the order of the coherence length. The amplitude of the induced spin polarization is approximately isotropic. This aspect is a consequence of the multicomponent $\mathbf{d}$ vector. A similar isotropic behavior is also observed for the case of longer-range penetration of the magnetization with a tendency to exhibit a sign change both in the TSC and in the FM side of the heterostructure [Figs. 3(e) and $3(\mathrm{f})]$.

\section{B. Magnetic proximity at the $\mathrm{Sr}_{2} \mathrm{RuO}_{4}-\mathrm{SrRuO}_{3}$ interface}

Here, as an experimentally relevant case to observe the inverse magnetic effects, we consider the $\mathrm{Sr}_{2} \mathrm{RuO}_{4}-\mathrm{SrRuO}_{3}$ heterostructure. $\mathrm{SrRuO}_{3}$ is an itinerant ferromagnet, which over the past years has been the subject of intense research [66-70]. By means of the density functional theory (DFT) approach (see Appendices), we demonstrate that the magnetic behavior of the $\mathrm{Sr}_{2} \mathrm{RuO}_{4}-\mathrm{SrRuO}_{3}$ heterostructure is nonstandard if compared to a conventional metal-ferromagnet interface and can lead to magnetization profiles resembling those that have been employed in the present study. Starting from the result that the bulk $\mathrm{Sr}_{2} \mathrm{RuO}_{4}$ is paramagnetic when the Coulomb repulsion $U_{214}$ is below about $0.7 \mathrm{eV}$, we find that for the interface layers proximized to $\mathrm{SrRuO}_{3}$, the Coulomb threshold for the transition into the ferromagnetic state is significantly reduced (see Appendices). Hence, to evaluate how the ground state of the $\mathrm{Sr}_{2} \mathrm{RuO}_{4}$ is affected by the ferromagnetism in $\mathrm{SrRuO}_{3}$, we investigate a superlattice made of five $\mathrm{RuO}_{2}$ layers in each side of the heterostructure [Fig. 4(a)]. We find that the ground state of the supercell is generally ferromagnetic in $\mathrm{SrRuO}_{3}$ with a magnetization that slightly decreases at the interface layers. $\mathrm{Sr}_{2} \mathrm{RuO}_{4}$ can also lower its energy by developing a nonvanishing magnetization that is collinear to that of the $\mathrm{SrRuO}_{3}$ close to the interface and can change sign away from it depending on the strength of the Coulomb interaction [Fig. 4(b)]. The interface layer always has the largest magnetization within $\mathrm{Sr}_{2} \mathrm{RuO}_{4}$. We tested several different magnetic orderings for the $\mathrm{SrRuO}_{3}-\mathrm{Sr}_{2} \mathrm{RuO}_{4}$ supercell, finding that the ferromagnetic coupling between $\mathrm{SrRuO}_{3}$ and $\mathrm{Sr}_{2} \mathrm{RuO}_{4}$ turns out to be the most stable for the supercell. For $U_{214}$ in the energy range [0.4-0.6] eV, we have a sign-changing magnetization in the layers of $\mathrm{Sr}_{2} \mathrm{RuO}_{4}$, while for $U_{214}=0.7 \mathrm{eV}$, all layers have a spin polarization which is aligned to that of the $\mathrm{SrRuO}_{3}$ and progressively decay in the $\mathrm{Sr}_{2} \mathrm{RuO}_{4}$ side of the heterostructure. The local magnetic moment increases with $U_{214}$, as expected, while the magnetic moment of $\mathrm{SrRuO}_{3}$ stays substantially unaltered. Although the computational time limits the size of the supercell which can be simulated, the resulting behavior provides clear trends of the way 


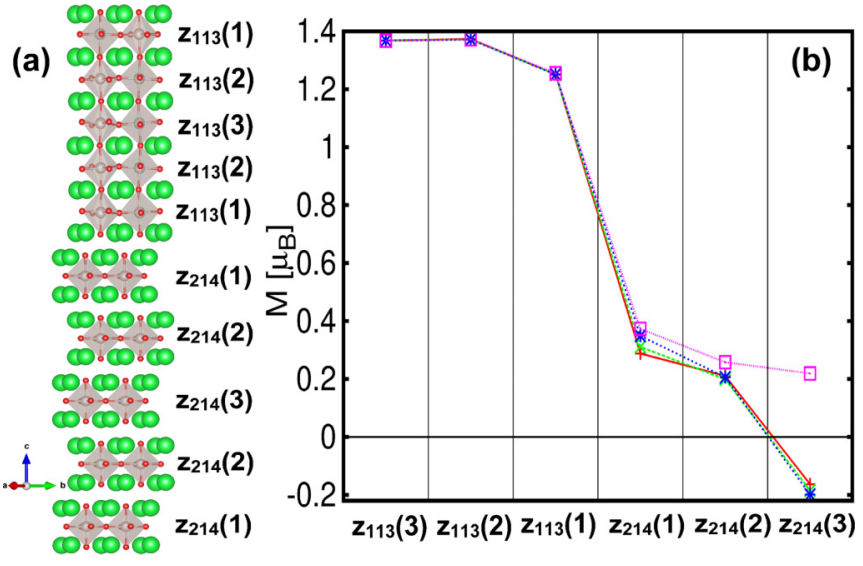

FIG. 4. (a) Schematic illustration of the $\mathrm{SrRuO}_{3}-\mathrm{Sr}_{2} \mathrm{RuO}_{4}$ superlattice with five $\mathrm{RuO}_{2}$ layers having cubic $\left(\mathrm{SrRuO}_{3}\right)$ and tetragonal $\left(\mathrm{Sr}_{2} \mathrm{RuO}_{4}\right)$ structure, respectively. (b) Evolution of the magnetization across the interface for various values of the Coulomb interaction $U_{214}$ in $\mathrm{Sr}_{2} \mathrm{RuO}_{4}$. Red, green, blue, and magenta points are for $U_{214}=0.4,0.5,0.6$, and $0.7 \mathrm{eV}$, respectively. $z_{113}(n)$ and $z_{214}(n)$ are the $n$th layer from the interface for the corresponding $\mathrm{Sr}_{2} \mathrm{RuO}_{4}$ and $\mathrm{SrRuO}_{3}$ sides of the superlattice.

magnetization gets reconstructed near the $\mathrm{SrRuO}_{3}-\mathrm{Sr}_{2} \mathrm{RuO}_{4}$ interface.

\section{DISCUSSION AND CONCLUSIONS}

We have demonstrated that inverse proximity effects in a TSC-FM heterostructure exhibit clear-cut signatures for both magnetic and superconducting interface reconstruction with respect to the nature of the pairing and the character of the magnetization leakage in the interface region of the TSC. The uniaxial d vector for the chiral TSC shows a magnetic response which is strongly anisotropic, with a tendency to a significant spatial modulation in amplitude and sign for the case of a long-range MP (Fig. 2). The helical TSC with planar d vector tends instead to yield a more isotropic spin polarization, except for the steplike magnetic profile, which is smaller (larger) in amplitude than that of the chiral state in the TSC (FM) side of the junction (Fig. 3). These features may be relevant to account for the observed anisotropy of the transport properties in $\mathrm{Sr}_{2} \mathrm{RuO}_{4}-\mathrm{SrRuO}_{3}[58,59]$. In such a system, by means of density functional approaches, we demonstrate that there occurs a magnetic instability near the interface for the electronic states of $\mathrm{Sr}_{2} \mathrm{RuO}_{4}$. This behavior is responsible for a longer-range penetration of the spin polarization into the normal metal with respect to the case of a conventional paramagnet. We argue that the tendency to undergo a changeover from paramagnetic to ferromagnetic in the $\mathrm{Sr}_{2} \mathrm{RuO}_{4}$ can set out the underlying scenario for the anomalous enhancement of the magnetic moments in a $\mathrm{Sr}_{2} \mathrm{RuO}_{4}-\mathrm{SrRuO}_{3}$ heterostructure [71]. Our results indicate that the presence of spin-triplet pairs can be accessed through magnetic measurements that are spatially resolved with respect to the TSC-FM interface and, for instance, performed across the superconducting transition.

In heterostructures based on conventional spin-singlet superconductors interfaced with itinerant ferromagnets, the magnetic field induced inside the superconductor has been experimentally observed by means of a variety of techniques such as nuclear magnetic resonance [72], Kerr effect [73], neutron scattering [74,75], and muon spin rotation measurements [76-78]. Since in the present work we are dealing with layered superconductors and we are interested in the short-range inverse proximity effects, polarized neutron reflectometry (PNR) is one of the most appropriate tools to investigate the layer-dependent magnetization profile $[79,80]$. Polarized neutron reflectometry is a highly sensitive technique, having measured the absolute magnetization of a monolayer of iron $\left(\sim 10^{-4} \mathrm{emu}\right)$, and has been successfully and largely employed to measure the layer-dependent magnetization profile of thin films and heterostructures with characteristic thickness from 2 to $5000 \AA$, exploiting the potential to detect the layer-resolved magnetic moment of the thin film. Preliminary measurements with PNR on heterostructures made of $\mathrm{SrRuO}_{3}$ thin films grown on the surface of $\mathrm{Sr}_{2} \mathrm{RuO}_{4}$ single crystals have been performed to detect the layer-dependent variation of the spin polarization above and below the critical superconducting temperature [81]. Hence, we expect that our results will be directly applicable and of significant impact for the ongoing debate about the nature of pairing in $\mathrm{Sr}_{2} \mathrm{RuO}_{4}$.

Another way to measure the magnetic field induced in the superconductor is by means of low-energy muon spectroscopy due to its extremely high sensitivity to magnetic field amplitude and its nanometer-depth resolution [76-78,82]. Indeed, $\mu \mathrm{SR}$ is able to detect small magnetic moments with amplitude $\sim 10^{-4}-10^{-5} \mu_{B}$ having high sensitivity over a relatively broader time window. This methodology has a lower degree of spatial sensitivity as compared to the polarized neutron reflectometry (it averages over regions of the order of $10 \mathrm{~nm}$ ). We expect that this type of spectroscopy can be employed in heterostructures of the type we have been investigating since the order of magnitude of the induced spin polarization is about $10^{-4} \mu_{B}$. Averaging of the spin polarization over several unit cells can lead to a tendency to cancel out the resulting magnetic effects for the helical pairing if compared to the chiral uniaxial d vector and this effect depends on the orientation of the magnetization and the character of the magnetic proximity.

Concerning the orbital effects associated with the action of the ferromagnet on the superconductor $[83,84]$ we observe that this mainly originates from Meissner screening currents flowing in the superconductor that produce magnetic fields decaying on distances that are of the order of the London penetration depth $(\lambda)$ and thus typically longer than the coherence length. The orbital effect is expected to be smaller than the spin one induced by the ferromagnet in the short-range scale close to the interface $[83,84]$; thus, on the length scale that we have considered in the presented study, it would be mostly negligible. Moreover, on the basis of the profile of the induced magnetization in the superconductor, one can qualitatively predict that the induced currents far from the interface will have a profile and behavior such as to screen the magnetic stray fields.

Finally, we comment on the role of disorder. In the present work, we are considering heterostructures with a high degree of structural and electronic matching being grown epitaxially. This type of fabrication method is typically employed 
to achieve hybrid materials with a significant control of the defects and of the level of atomic interdiffusion or contamination at the interface. On the other hand, for disordered interfaces, odd-parity $p$-wave superconductivity is expected to exhibit a significant suppression of the superconducting order parameter. Thus, one would not have a spatial domain close to the interface with a substantial coexistence of nonvanishing magnetization and spin-triplet superconducting order parameter. In this scenario, it is plausible to expect that the inverse proximity effect into the superconductor will be weak. Instead, there might be an induced spin polarization at the interface through the nonmagnetic region due to the leakage of the spin-triplet Cooper pairs into the ferromagnet. Taking into account the performed analysis, we observe that in this case, one realizes a sort of superconductor-normal-ferromagnet heterostructure. Thus, there would be a weak interplay between superconductivity and ferromagnetism as they are substantially spatially separated.

\section{ACKNOWLEDGMENTS}

O.M. and C.A. contributed equally to this work. C.A. is supported by the Foundation for Polish Science through the International Research Agendas program co-financed by the European Union within the Smart Growth Operational Programme. We acknowledge the CINECA award under the ISCRA initiatives IsC76 "MEPBI" and IsC81 "DISTANCE" Grant, for the availability of high-performance computing resources and support. M.C. and P.G. acknowledge support from the project "Two-dimensional Oxides Platform for SPIN-orbitronics nanotechnology (TOPSPIN)" funded by the MIUR-PRIN Bando 2017, Grant No. 20177SL7HC.

\section{APPENDIX A: EFFECTIVE MODEL FOR MAGNETIC PROXIMITY IN THE SPIN-TRIPLET SUPERCONDUCTOR}

In this section, we compare the impact of the exchange field on both monolayer and bilayer geometries with chiral and helical spin-triplet superconductivity in order to evaluate, in a simplified configuration, the local role of an inhomogeneous magnetic proximity close to the interface.

\section{Superconducting spin polarization vs exchange field for monolayer}

Let us start by considering a monolayer spin-triplet superconductor in the presence of a Zeeman term that mimics the induced magnetic proximity. We investigate the behavior of the normal-state magnetization $M^{\mathrm{N}}$ compared with the spin polarization $M^{\mathrm{SC}}$ induced by the magnetic exchange in the superconducting state. This analysis allows to understand how the induced spin polarization evolves in the spin-triplet superconductor.

In the case of chiral superconductivity, the $\vec{d}$ vector is uniaxial and pointing along the out-of-plane direction with respect to the electron motion. This means that Cooper pairs have $S_{z}=0$, and the equal spin configurations are generically aligned in the $x-y$ plane. The outcome of the spin polarization in the superconducting state is reported in Tables I and II. We find that the induced spin moments are always aligned to those of the normal-state magnetization and, as expected, there is a
TABLE I. Summary of the induced magnetization $M^{\mathrm{SC}}$ for a monolayer with chiral superconductivity. Results are for two directions of the magnetic exchange field in three different regimes of amplitude for $\vec{h}$.

\begin{tabular}{lcc}
\hline \hline & $\vec{h} \| \vec{d}$ & $\vec{h} \perp \vec{d}$ \\
\hline$\vec{h} / \Delta_{0}$ & $M^{\mathrm{SC}}$ & $M^{\mathrm{SC}}$ \\
(a) $\vec{h}<\Delta$ & 0 & $0.9 M^{\mathrm{N}}$ \\
(b) $\vec{h} \approx \Delta$ & 0 & $M^{\mathrm{N}}$ \\
(c) $\vec{h}>\Delta$ & $M^{\mathrm{N}}$ & $M^{\mathrm{N}}$ \\
\hline \hline
\end{tabular}

tendency to have a vanishing spin polarization with the field parallel to the $\vec{d}$ vector upon reaching an amplitude that is comparable to the superconducting gap. Instead, if we have a configuration of $\vec{h}$ perpendicular to the $\vec{d}$ vector, then there is always a net spin polarization due to the Cooper pair spin moments being aligned to $\vec{h}$. Hence, Cooper pairs can get spin polarized without pair breaking and the magnetization has a typical amplitude that is close to that of the normal state.

In the case of a helical superconductor, the $\vec{d}$ vector has a planar orientation that changes in the momentum space. This means that there are Cooper pairs with both spin projections $S_{x}=0$ and $S_{y}=0$. This results in a superconducting order parameter with Cooper pairs that are spin polarized along the $z$ direction. Starting with an exchange field that is transverse to the $\vec{d}$ vector, we have that the spin moment of the Cooper pairs is aligned to $\vec{h}$. Hence, we obtain $M^{\mathrm{SC}}=M^{\mathrm{N}}$ as it was for the chiral spin-triplet pairing. The case with $h_{x}$ is richer in terms of possible outcomes. For this configuration, there are $\vec{k}$ points in the reciprocal space for which one gets $\vec{h}$ parallel to $\vec{d}$, while for others, $\vec{h}$ is noncollinear to $\vec{d}$. Indeed, one has an angle between the field and the superconducting $\vec{d}$ vector that can vary between 0 and $\pi$. Then, for $h_{x}<\Delta$, one can only spin polarize a portion of spin triplet pairs and, consequently, $M^{\mathrm{SC}}<M^{\mathrm{N}}$. Additionally, by allowing a configuration of the order parameter with $C_{4}$ rotational symmetry breaking, we obtain that the superconductor can gain energy by having more electron pairs polarized at the expense of redistributing them to the Fermi surface, making the order parameter asymmetric, i.e., $d_{x} \neq d_{y}$. In this case, we obtain an increase of the magnetization, $M^{\mathrm{SC}^{*}}>M^{\mathrm{SC}}$. The resulting findings are summarized in Table II.

TABLE II. Summary of the induced magnetization $M^{\mathrm{SC}}$ for a monolayer with helical superconductivity. Results are for two directions of the magnetic exchange field in three different regimes of amplitude for $\vec{h}$ with respect to the superconducting gap energy $\Delta$, i.e., $\vec{h}<\Delta, \vec{h} \approx \Delta$, and $\vec{h}>\Delta$, respectively.

\begin{tabular}{lccc}
\hline \hline & \multicolumn{2}{c}{$\vec{h}$ coplanar $\vec{d}(\vec{k})$} & $\vec{h} \perp \vec{d}$ \\
\hline$\vec{h} / \Delta_{0}$ & $M^{\mathrm{SC}}$ & $M^{\mathrm{SC} *}$ & $M^{\mathrm{SC}}$ \\
(a) $\vec{h}<\Delta$ & $0.45 M^{\mathrm{N}}$ & $0.46 M^{\mathrm{N}}$ & $M^{\mathrm{N}}$ \\
(b) $\vec{h} \approx \Delta$ & $0.56 M^{\mathrm{N}}$ & $0.70 M^{\mathrm{N}}$ & $M^{\mathrm{N}}$ \\
(c) $\vec{h}>\Delta$ & $M^{\mathrm{N}}$ & $M^{\mathrm{N}}$ & $M^{\mathrm{N}}$ \\
\hline \hline
\end{tabular}


TABLE III. Summary of the results for the bilayer with chiral superconductivity.

\begin{tabular}{lcccc}
\hline \hline Case & $\begin{array}{c}\text { Configurations } \\
\left(\frac{h_{1}}{\Delta_{0}}, \frac{h_{2}}{\Delta_{0}}\right)\end{array}$ & $\left(\frac{\Delta_{1}}{\Delta_{0}}, \frac{\Delta_{2}}{\Delta_{0}}\right)$ & $\begin{array}{c}\vec{h} \| \vec{d} \\
\left(\frac{M_{1}}{M^{\mathrm{N}}}, \frac{M_{2}}{M^{\mathrm{N}}}\right)\end{array}$ & $\begin{array}{c}\vec{h} \perp \vec{d} \\
\left(\frac{M_{1}}{M^{\mathrm{N}}}, \frac{M_{2}}{M^{\mathrm{N}}}\right)\end{array}$ \\
\hline (a) & $(0.6,0.6)$ & $(1,1)$ & $(0,0)$ & $(1,1)$ \\
(b) & $(0.4,0.6)$ & $(1,1)$ & $(-0.2,0.15)$ & $(0.98,0.99)$ \\
(c) & $(0.2,0.6)$ & $(1,1)$ & $(-0.8,0.3)$ & $(0.80,0.92)$ \\
(d) & $(0.1,5)$ & $(1,0)$ & $(0.65,1)$ & $(1.03,1)$ \\
(e) & $(0.1,20)$ & $(1,0)$ & $(1.5,1)$ & $(0.96,1)$ \\
(f) & $(0.1,5)$ & $(1,0.2)$ & $(0.53,0.98)$ & $(1.07,1)$ \\
\hline \hline
\end{tabular}

\section{Layer-dependent magnetic exchange}

Let us consider a bilayer geometry which is the minimal configuration that allows us to investigate the consequence of a nonuniform magnetic exchange field. Having two distinct layers, one can introduce a layer-dependent exchange that can locally mimic the behavior of the induced spin polarization in a given heterostructure in close proximity to the interface. Hence, we consider both layers to be superconducting and the exchange magnetic fields have amplitude $h_{1}$ and $h_{2}$, respectively. The energy scale of the superconducting order parameters is $\Delta_{1(2)}$. Here, we focus again on the induced magnetic response in each layer by evaluating the spin polarization for the ground state.

First, we discuss the regime of small exchange amplitude [see rows (a)-(c) in Tables III and IV] and uniform $\Delta$. These regimes are appropriate for a magnetic proximity with the magnetization penetrating inside the superconductor, with the gap keeping about its bulk value. In the case of uniform $h$, the results substantially reproduce the effects of the single-layer system considered above. In fact, for $\vec{h} \perp \vec{d}$ in both the chiral and helical configurations, we obtain $M^{\mathrm{SC}} \approx$ $M^{\mathrm{N}}$. Interestingly, when the exchange field is not uniform, instead of following the $\vec{h}(z)$ profile, the magnetization gets smoothed out, such that it has a smaller gradient compared to that in the normal state. This can be qualitatively ascribed to the fact that Cooper pairs can have a larger interlayer tunneling probability compared to the single electrons in the normal state. Another nonstandard behavior is obtained in the regime of strong gradient and large amplitude of the exchange field. There, the magnetization can have an amplitude that is even greater than that of the normal state, as reported in rows

TABLE IV. Summary of the results for the bilayer with helical superconductivity.

\begin{tabular}{lcccc}
\hline \hline Case & $\begin{array}{c}\text { Configurations } \\
\left(\frac{h_{1}}{\Delta_{0}}, \frac{h_{2}}{\Delta_{0}}\right)\end{array}$ & $\left(\frac{\Delta_{1}}{\Delta_{0}}, \frac{\Delta_{2}}{\Delta_{0}}\right)$ & $\begin{array}{c}\vec{h} \text { coplanar } \vec{d} \\
\left(\frac{M_{1}}{M^{\mathrm{N}}}, \frac{M_{2}}{M^{\mathrm{N}}}\right)\end{array}$ & $\begin{array}{c}\vec{h} \perp \vec{d} \\
\left(\frac{M_{1}}{M^{\mathrm{N}}}, \frac{M_{2}}{M^{\mathrm{N}}}\right)\end{array}$ \\
\hline (a) & $(0.6,0.6)$ & $(1,1)$ & $(0.55,0.55)$ & $(1,1)$ \\
(b) & $(0.4,0.6)$ & $(1,1)$ & $(0.43,0.60)$ & $(0.98,0.99)$ \\
(c) & $(0.2,0.6)$ & $(1,1)$ & $(0 ., 0.63)$ & $(0.80,0.92)$ \\
(d) & $(0.1,5)$ & $(1,0)$ & $(0.94,1)$ & $(1.03,1)$ \\
(e) & $(0.1,20)$ & $(1,0)$ & $(1.19,1)$ & $(0.96,1)$ \\
(f) & $(0.1,5)$ & $1,0.2$ & $(0.84,1)$ & $(1.07,1)$ \\
\hline \hline
\end{tabular}

(d)-(f) for $\vec{h} \perp \vec{d}$ in Tables III and IV. This implies that a change in the number of electron pairs is in place when $\vec{h}$ is larger than $\Delta$, which allows one to have an enhancement of the spin polarization.

Next, we consider the configuration $\vec{h} \| \vec{d}$. The first relevant difference with the previous case is that a variation of the exchange field can lead to a spin polarization with opposite signs in the two layers. This behavior is reminiscent of the oscillation of the magnetization found in the multilayer system and generally indicates a tendency of the spin-triplet superconductor to exhibit a diamagnetic response when the exchange field is nonuniform. The helical case, however, never shows a sign change, setting a clear difference between a $\vec{d}$ vector lying in a plane or with an axial configuration. Since in the weak gradient regime [row (c) of Table IV], one can reach a vanishing value of the spin polarization, it is plausible to expect that a weak sign-changing profile of the spin polarization can be obtained in a multilayered configuration.

This trend is consistent with the observation of a more pronounced enhancement of $M^{\mathrm{SC}}-M^{\mathrm{N}}$ in the chiral spin-triplet superconductor.

\section{APPENDIX B: DENSITY FUNCTIONAL THEORY ANALYSIS}

In this section, we provide the details of the $a b$ initio computation with the evolution of the magnetic instability in $\mathrm{Sr}_{2} \mathrm{RuO}_{4}$ as a function of Coulomb interaction and strain.

\section{Computational details}

We perform spin-polarized first-principles density functional calculations within the local spin density approximation (LSDA) [85,86] by using the plane-wave VASP [87] DFT package and the Perdew-Zunger [88] parametrization of the Ceperley-Alder data [89] for the exchange-correlation functional. The choice of the LSDA exchange functional is suggested by a paper [90] where the generalized gradient approximation (GGA) is shown to be less accurate than LSDA for $\mathrm{SrRuO}_{3}$. The core and the valence electrons were treated within the projector augmented wave (PAW) method [91] and with a cutoff of $430 \mathrm{eV}$ for the plane-wave basis. An $8 \times 8 \times 1 k$-point grid is used for the full relaxation of the bulk orthorhombic phase with spin-orbit coupling. We use a $10 \times 10 \times 1 k$-point grid centered at the $\Gamma$ point for the determination of the total energy of $\mathrm{Sr}_{2} \mathrm{RuO}_{4}$ and the full relaxation of the heterostructure. In all cases, the tetrahedron method with Blöchl corrections [92,93] was used for the Brillouin zone integrations. We optimize the internal degrees of freedom by minimizing the total energy to be less than $10^{-5} \mathrm{eV}$ and the remaining forces to be less than $5 \mathrm{meV} / \AA$.

To catch the magnetic behavior, the Hubbard $U$ effects at the $\mathrm{Ru}$ sites for $4 d$ orbitals were included in the LSDA $+U$ [94,95] approach using the rotationally invariant scheme proposed in Ref. [96] and implemented in VASP [87]. We denote by $U_{113}$ and $J_{H, 113}$ the Coulomb repulsion and the Hund parameter of $\mathrm{SrRuO}_{3}$, while $U_{214}$ and $J_{H, 214}$ are for $\mathrm{Sr}_{2} \mathrm{RuO}_{4}$. In this study, we fixed $U_{113}=1 \mathrm{eV}$ for the $\mathrm{Ru} 4 d$ states of $\mathrm{SrRuO}_{3}$, while we use $J_{H, 113}=0.15 U_{113}$. We keep $J_{H, 214}=0$ and we tune from 0 to $1.5 \mathrm{eV}$ the Coulomb repulsion in 




FIG. 5. Magnetic phase diagram of bulk $\mathrm{Sr}_{2} \mathrm{RuO}_{4}$ as a function of the Coulomb repulsion and in-plane strain.

$\mathrm{Sr}_{2} \mathrm{RuO}_{4}$. These values are common to all the supercell calculations presented in this work.

The study of $\mathrm{SrRuO}_{3}$ under strain is made with the in-plane lattice constant of $\mathrm{Sr}_{2} \mathrm{RuO}_{4}$. A small value of $U$ was used in the bulk to reproduce the correct magnetization in DFT, but in extreme strain conditions, one does not have a reference of $U$ to describe the corresponding physical condition.

In order to simulate thin films of $\mathrm{SrRuO}_{3}$ grown on a $\mathrm{Sr}_{2} \mathrm{RuO}_{4}$ substrate, we fix the in-plane lattice parameter $a$ with the experimental value of the $\mathrm{Sr}_{2} \mathrm{RuO}_{4}$ bulk, and the atomic positions of the $\mathrm{Sr}_{2} \mathrm{RuO}_{4}$ phase are fixed to the bulk values. We use the value of $c$ such that the volume of $\mathrm{SrRuO}_{3}$ is equal to the bulk volume because the theoretical volume underestimates the experimental one. We discuss the structural and electronic properties of heterostructures made of $\mathrm{Sr}_{2} \mathrm{RuO}_{4}$ and $\mathrm{SrRuO}_{3}$. We will investigate the supercell composed of three $\mathrm{Sr}_{2} \mathrm{RuO}_{4}$ and five $\mathrm{SrRuO}_{3}$ layers. The focus is on the superlattice structures with three inequivalent layers of $\mathrm{Sr}_{2} \mathrm{RuO}_{4}$ and three inequivalent layers of $\mathrm{SrRuO}_{3}$. Being the magnetic order in $\mathrm{SrRuO}_{3}$ dominating, we first study the magnetic order in the $\mathrm{SrRuO}_{3}$ side of the heterostructure, fixing $U_{214}=0$. After that, we add the Coulomb repulsion in $\mathrm{Sr}_{2} \mathrm{RuO}_{4}$ to investigate the magnetic instability of the $\mathrm{Sr}_{2} \mathrm{RuO}_{4}$ side of the supercell. Structural optimization was performed in the LSDA $+U$ approximation separately for the different magnetic cases.

\section{Magnetic instability in $\mathrm{Sr}_{2} \mathrm{RuO}_{4}$}

We discuss the role of the Coulomb interaction and the strain in the $\mathrm{Sr}_{2} \mathrm{RuO}_{4}$ system together with the possible magnetic instabilities and their character.

The $\mathrm{Sr}_{2} \mathrm{RuO}_{4}$ compound is paramagnetic in the bulk. We compare the energy of the ferromagnetic (FM), paramagnetic (PM), and antiferromagnetic (AFM) phases in terms of $U_{214}$ from 0 to $1 \mathrm{eV}$ and by varying the in-plane lattice constant from $-3 \%$ to $+3 \%$. The outcome is reported in Fig. 5. The AFM phase is G-type, as it can also be found in the ultrathin $\mathrm{SrRuO}_{3}$. The system is paramagnetic for small values of $U$ and it is close to both the AFM and FM magnetic phases for compressive and tensile strain, respectively.

The rotations of the octahedra are not stabilized by the strain $[97,98]$. Because there are no rotations, the most


FIG. 6. Energy as a function of the magnetic moment of the bulk $\mathrm{Sr}_{2} \mathrm{RuO}_{4}$ for different values of the Coulomb repulsion $U$. Top panel: The presence of the ferromagnetic minimum close to the paramagnetic minimum thanks to the Van Hove singularity (VHS) in the $d_{x y}$ band. Bottom panel: A first-order transition between two ferromagnetic minima thanks to the VHS in the $d_{\gamma z}$ bands. The energy of the paramagnetic phase is the reference point.

important effect is the dependence of the hopping parameters on the Ru-Ru distance. When the lattice constant gets larger, the hopping decreases and the role of electron correlations becomes more relevant. This explains the magnetic instability at larger in-plane lattice constant $(a)$ as a function of $U$. If we impose the rotations of the octahedra and the consequent reduction of the bandwidth, there is a critical value of the rotation beyond which $\mathrm{Sr}_{2} \mathrm{RuO}_{4}$ becomes ferromagnetic at any small value of the Coulomb interaction. The magnetic properties in Fig. 5 do not change qualitatively if we include spin-orbit coupling or by assuming that the atomic positions are not relaxed.

Now, we fix the atomic positions to the experimental values and the volume to more accurately study the transition from the PM to the FM phase.

We calculate in detail the total energy as a function of the magnetization in Fig. 6 for the values of $U$ close to the magnetic transition. In the top panel, we can observe the double minima curve giving rise to a first-order magnetic transition that is typical in a metamagnetic system. The spin-polarized state becomes lower in energy when $U$ increases and becomes the ground state at $U=0.61 \mathrm{eV}$. 
[1] M. Sigrist and K. Ueda, Phenomenological theory of unconventional superconductivity, Rev. Mod. Phys. 63, 239 (1991).

[2] Y. Tanaka, T. Hirai, K. Kusakabe, and S. Kashiwaya, Theory of the Josephson effect in a superconductor/one-dimensional electron gas/superconductor junction, Phys. Rev. B 60, 6308 (1999).

[3] N. Read and D. Green, Paired states of fermions in two dimensions with breaking of parity and time-reversal symmetries, and the fractional quantum Hall effect, Phys. Rev. B 61, 10267 (2000).

[4] D. A. Ivanov, Non-Abelian Statistics of Half-Quantum Vortices in P-Wave Superconductors, Phys. Rev. Lett. 86, 268 (2001).

[5] A. Y. Kitaev, Unpaired Majorana fermions in quantum wires, Phys. Usp. 44, 131 (2001).

[6] G. E. Volovik, The Universe in a Helium Droplet (Oxford University Press, Oxford, 2003).

[7] Y. Maeno, S. Kittaka, T. Nomura, S. Yonezawa, and K. Ishida, Evaluation of spin-triplet superconductivity in $\mathrm{Sr}_{2} \mathrm{RuO}_{4}$, J. Phys. Soc. Jpn. 81, 011009 (2012).

[8] M. Sato and Y. Ando, Topological superconductors: A review, Rep. Prog. Phys. 80, 076501 (2017).

[9] J. Spałek, Spin-triplet superconducting pairing due to local Hund's rule and Dirac exchange, Phys. Rev. B 63, 104513 (2001).

[10] C. M. Puetter and H.-Y. Kee, Identifying spin-triplet pairing in spin-orbit coupled multi-band superconductors, Europhys. Lett. 98, 27010 (2012).

[11] J. E. Han, Spin-triplet s-wave local pairing induced by Hund's rule coupling, Phys. Rev. B 70, 054513 (2004).

[12] Y. Fukaya, S. Tamura, K. Yada, Y. Tanaka, P. Gentile, and M. Cuoco, Interorbital topological superconductivity in spin-orbit coupled superconductors with inversion symmetry breaking, Phys. Rev. B 97, 174522 (2018).

[13] H. G. Suh, H. Menke, P. M. Brydon, C. Timm, A. Ramires, and D. F. Agterberg, Stabilizing even-parity chiral superconductivity in $\mathrm{Sr}_{2} \mathrm{RuO}_{4}$, Phys. Rev. Research 2, 032023(R) (2020).

[14] S. Murakami, N. Nagaosa, and M. Sigrist, An SO(5) Model of P-Wave Superconductivity and Ferromagnetism, Phys. Rev. Lett. 82, 2939 (1999).

[15] E. Dumitrescu and S. Tewari, Topological properties of the time-reversal-symmetric Kitaev chain and applications to organic superconductors, Phys. Rev. B 88, 220505(R) (2013).

[16] E. Dumitrescu, J. D. Sau, and S. Tewari, Magnetic field response and chiral symmetry of time-reversal-invariant topological superconductors, Phys. Rev. B 90, 245438 (2014).

[17] T. Hyart, A. R. Wright, and B. Rosenow, Zeeman-field induced topological phase transitions in triplet superconductors, Phys. Rev. B 90, 064507 (2014).

[18] M. T. Mercaldo, M. Cuoco, and P. Kotetes, Magnetic-fieldinduced topological reorganization of a $p$-wave superconductor, Phys. Rev. B 94, 140503(R) (2016).

[19] M. T. Mercaldo, M. Cuoco, and P. Kotetes, Magnetic manipulation of topological states in $p$-wave superconductors, Physica B 536, 730 (2018).

[20] M. T. Mercaldo, P. Kotetes, and M. Cuoco, Topological signatures of the coexistence of antiferromagnetism and oddparity spin-triplet superconductivity, AIP Adv. 8, 101303 (2018).

[21] K. Sengupta, I. Žutić, H.-J. Kwon, V. M. Yakovenko, and S. Das Sarma, Midgap edge states and pairing symmetry of quasi-one- dimensional organic superconductors, Phys. Rev. B 63, 144531 (2001).

[22] H.-J. Kwon, K. Sengupta, and V. M. Yakovenko, Fractional AC Josephson effect in unconventional superconductors, Low Temp. Phys. 30, 613 (2004).

[23] K. Sengupta and V. M. Yakovenko, Spontaneous Spin Accumulation in Singlet-Triplet Josephson Junctions, Phys. Rev. Lett. 101, 187003 (2008).

[24] M. Cuoco, A. Romano, C. Noce, and P. Gentile, Proximity effect between an unconventional superconductor and a ferromagnet with spin bandwidth asymmetry, Phys. Rev. B 78, 054503 (2008).

[25] A. Romano, M. Cuoco, C. Noce, P. Gentile, and G. Annunziata, Field-induced transition from chiral spin-triplet to mixed-parity Fulde-Ferrell-Larkin-Ovchinnikov superconductivity, Phys. Rev. B 81, 064513 (2010).

[26] P. M. R. Brydon, C. Iniotakis, D. Manske, and M. Sigrist, Functional Superconductor Interfaces from Broken Time-Reversal Symmetry, Phys. Rev. Lett. 104, 197001 (2010).

[27] P. Gentile, M. Cuoco, A. Romano, C. Noce, D. Manske, and P. M. R. Brydon, Spin-Orbital Coupling in a Triplet Superconductor-Ferromagnet Junction, Phys. Rev. Lett. 111, 097003 (2013).

[28] P. M. R. Brydon, Y. Asano, and C. Timm, Spin Josephson effect with a single superconductor, Phys. Rev. B 83, 180504(R) (2011).

[29] P. M. R. Brydon, W. Chen, Y. Asano, and D. Manske, Charge and spin supercurrents in triplet superconductor-ferromagnetsinglet superconductor Josephson junctions, Phys. Rev. B 88, 054509 (2013).

[30] J. Linder and J. W. A. Robinson, Superconducting spintronics, Nat. Phys. 11, 307 (2015).

[31] A. Yu. Kitaev, Fault-tolerant quantum computation by anyons, Ann. Phys. 303, 2 (2003).

[32] C. Nayak, S. H. Simon, A. Stern, M. Freedman, and S. Das Sarma, Non-Abelian anyons and topological quantum computation, Rev. Mod. Phys. 80, 1083 (2008).

[33] J. Alicea, Y. Oreg, G. Refael, F. von Oppen, and M. P. A. Fisher, Non-Abelian statistics and topological quantum information processing in 1D wire networks, Nat. Phys. 7, 412 (2011).

[34] G. R. Stewart, Z. Fisk, J. O. Willis, and J. L. Smith, Possibility of Coexistence of Bulk Superconductivity and Spin Fluctuations in $\mathrm{UPt}_{3}$, Phys. Rev. Lett. 52, 679 (1984).

[35] S. S. Saxena, P. Agarwal, K. Ahilan, F. M. Grosche, R. K. W. Haselwimmer, M. J. Steiner, E. Pugh, I. R. Walker, S. R. Julian, P. Monthoux, G. G. Lonzarich, A. Huxley, I. Sheikin, D. Braithwaite, and J. Flouquet, Superconductivity on the border of itinerant-electron ferromagnetism in $\mathrm{UGe}_{2}$, Nature (London) 406, 587 (2000).

[36] M. Kyogaku, Y. Kitaoka, K. Asayama, C. Geibel, C. Schank, and F. Steglich, NMR and NQR studies of magnetism and superconductivity in the antiferromagnetic heavy fermion superconductors $\mathrm{UM}_{2} \mathrm{Al}_{3}(\mathrm{M}=\mathrm{Ni}$ and Pd), J. Phys. Soc. Jpn. 62, 4016 (1993).

[37] L. Jiao, S. Howard, S. Ran, Z. Wang, J. O. Rodriguez, M. Sigrist, Z. Wang, N. P. Butch, and V. Madhavan, Chiral superconductivity in heavy-fermion metal $\mathrm{UTe}_{2}$, Nature (London) 579, 523 (2020).

[38] S. Ran, C. Eckberg, Q.-P. Ding, Y. Furukawa, T. Metz, S. R. Saha, I.-L. Liu, M. Zic, H. Kim, J. Paglione, and N. P. Butch, 
Nearly ferromagnetic spin-triplet superconductivity, Science 365, 684 (2019).

[39] E. Bauer, G. Hilscher, H. Michor, C. Paul, E. W. Scheidt, A. Gribanov, Y. Seropegin, H. Noël, M. Sigrist, and P. Rogl, Heavy Fermion Superconductivity and Magnetic Order in Noncentrosymmetric $\mathrm{CePt}_{3} \mathrm{Si}$, Phys. Rev. Lett. 92, 027003 (2004).

[40] M. Nishiyama, Y. Inada, and G.-q. Zheng, Spin Triplet Superconducting State due to Broken Inversion Symmetry in $\mathrm{Li}_{2} \mathrm{Pt}_{3} \mathrm{~B}$, Phys. Rev. Lett. 98, 047002 (2007).

[41] A. G. Lebed, K. Machida, and M. Ozaki, Triplet electron pairing and anisotropic spin susceptibility in organic superconductors (TMTSF) $)_{2}$ X, Phys. Rev. B 62, R795(R) (2000).

[42] I. J. Lee, S. E. Brown, W. G. Clark, M. J. Strouse, M. J. Naughton, W. Kang, and P. M. Chaikin, Triplet Superconductivity in an Organic Superconductor Probed by NMR Knight Shift, Phys. Rev. Lett. 88, 017004 (2001).

[43] J. Shinagawa, Y. Kurosaki, F. Zhang, C. Parker, S. E. Brown, D. Jérome, J. B. Christensen, and K. Bechgaard, Superconducting State of the Organic Conductor (TMTSF) ${ }_{2} \mathrm{ClO}_{4}$, Phys. Rev. Lett. 98, 147002 (2007).

[44] K. Matano, M. Kriener, K. Segawa, Y. Ando, and G.-q. Zheng, Spin-rotation symmetry breaking in the superconducting state of $\mathrm{Cu}_{x} \mathrm{Bi}_{2} \mathrm{Se}_{3}$, Nat. Phys. 12, 852 (2016).

[45] J.-K. Bao, J.-Y. Liu, C.-W. Ma, Z.-H. Meng, Z.-T. Tang, Y.-L. Sun, H.-F. Zhai, H. Jiang, H. Bai, C.-M. Feng, Z.-A. Xu, and G.-H. Cao, Superconductivity in Quasi-One-Dimensional $\mathrm{K}_{2} \mathrm{Cr}_{3} \mathrm{As}_{3}$ with Significant Electron Correlations, Phys. Rev. X 5, 011013 (2015).

[46] G. Cuono, C. Autieri, F. Forte, M. T. Mercaldo, A. Romano, A. Avella, and C. Noce, A minimal tight-binding model for the quasi-one-dimensional superconductor $\mathrm{K}_{2} \mathrm{Cr}_{3} \mathrm{As}_{3}$, New J. Phys. 21, 063027 (2019).

[47] C. Noce, The chromium pnictide materials: A tunable platform for exploring new exciting phenomena, Europhys. Lett. 130, 67001 (2020).

[48] A. Pustogow, Y. Luo, A. Chronister, Y. S. Su, D. A. Sokolov, F. Jerzembeck, A. P. Mackenzie, C. W. Hicks, N. Kikugawa, S. Raghu, E. D. Bauer, and S. E. Brown, Pronounced drop of ${ }^{17} \mathrm{O}$ NMR Knight shift in superconducting state of $\mathrm{Sr}_{2} \mathrm{RuO}_{4}$, Nature (London) 574, 72 (2019).

[49] K. Ishida, M. Manago, and Y. Maeno, Horizontal line nodes in $\mathrm{Sr}_{2} \mathrm{RuO}_{4}$ proved by spin resonance, J. Phys. Soc. Jpn. 89, 034712 (2020).

[50] A. N. Petsch, M. Zhu, M. Enderle, Z. Q. Mao, Y. Maeno, I. I. Mazin, and S. M. Hayden, Reduction of the Spin Susceptibility in the Superconducting State of $\mathrm{Sr}_{2} \mathrm{RuO}_{4}$ Observed by Polarized Neutron Scattering, Phys. Rev. Lett. 125, 217004 (2020).

[51] A. P. Mackenzie, T. Scaffidi, C. W. Hicks, and Y. Maeno, Even odder after twenty-three years: The superconducting order parameter puzzle of $\mathrm{Sr}_{2} \mathrm{RuO}_{4}$, npj Quantum Mater. 2, 40 (2017).

[52] T. Tokuyasu, J. A. Sauls, and D. Rainer, Proximity effect of a ferromagnetic insulator in contact with a superconductor, Phys. Rev. B 38, 8823 (1988)

[53] F. S. Bergeret, A. F. Volkov, and K. B. Efetov, Induced ferromagnetism due to superconductivity in superconductorferromagnet structures, Phys. Rev. B 69, 174504 (2004).

[54] S. Tollis, M. Daumens, and A. Buzdin, Inversion of the proximity effect in atomic-scale ferromagnet/superconductor/ ferromagnet trilayers, Phys. Rev. B 71, 024510 (2005).
[55] F. S. Bergeret, A. Levy Yeyati, and A. Martin-Rodero, Inverse proximity effect in superconductor-ferromagnet structures: From the ballistic to the diffusive limit, Phys. Rev. B 72, 064524 (2005).

[56] A. Di Bernardo, S. Komori, G. Livanas, G. Divitini, P. Gentile, M. Cuoco, and J. W. A. Robinson, Nodal superconducting exchange coupling, Nat. Mater. 18, 1194 (2019).

[57] M. S. Anwar et al., Direct penetration of spin-triplet superconductivity into a ferromagnet in $\mathrm{Au} / \mathrm{SrRuO}_{3} / \mathrm{Sr}_{2} \mathrm{RuO}_{4}$ junctions, Nat. Commun. 7, 13220 (2016).

[58] M. S. Anwar, M. Kunieda, R. Ishiguro, S. R. Lee, L. A. B. Olde Olthof, J. W. A. Robinson, S. Yonezawa, T. W. Noh, and Y. Maeno, Observation of superconducting gap spectra of longrange proximity effect in $\mathrm{Au} / \mathrm{SrTiO}_{3} / \mathrm{SrRuO}_{3} / \mathrm{Sr}_{2} \mathrm{RuO}_{4}$ tunnel junctions, Phys. Rev. B 100, 024516 (2019).

[59] M. S. Anwar, M. Kunieda, R. Ishiguro, S. R. Lee, C. Sow, J. W. A. Robinson, S. Yonezawa, T. W. Noh, and Y. Maeno, Anomalous anisotropic behaviour of spin-triplet proximity effect in $\mathrm{Au} / \mathrm{SrRuO}_{3} / \mathrm{Sr}_{2} \mathrm{RuO}_{4}$ junctions, Sci. Rep. 9, 15827 (2019).

[60] Y. Krockenberger et al., Growth of superconducting $\mathrm{Sr}_{2} \mathrm{RuO}_{4}$ thin films, Appl. Phys. Lett. 97, 082502 (2010).

[61] P. B. Marshall, H. Kim, K. Ahadi, and S. Stemmer, Growth of strontium ruthenate films by hybrid molecular beam epitaxy, APL Mater. 5, 096101 (2017).

[62] M. Uchida et al., Molecular beam epitaxy growth of superconducting $\mathrm{Sr}_{2} \mathrm{RuO}_{4}$ films, APL Mater. 5, 106108 (2017).

[63] C. M. Palomares Garcia et al., Pair suppression caused by mosaic-twist defects in superconducting $\mathrm{Sr}_{2} \mathrm{RuO}_{4}$ thin-films prepared using pulsed laser deposition, Commun. Mater. 1, 23 (2020).

[64] D. Terrade, D. Manske, and M. Cuoco, Control of edge currents at a ferromagnet - triplet superconductor interface by multiple helical Majorana modes, Phys. Rev. B 93, 104523 (2016).

[65] D. Terrade, P. Gentile, M. Cuoco, and D. Manske, Proximity effects in spin-triplet superconductor-ferromagnet heterostucture with spin-active interface, Phys. Rev. B 88, 054516 (2013).

[66] J. Matsuno, N. Ogawa, K. Yasuda, F. Kagawa, W. Koshibae, N. Nagaosa, Y. Tokura, and M. Kawasaki, Interface-driven topological Hall effect in $\mathrm{SrRuO}_{3}-\mathrm{SrIrO}_{3}$ bilayer, Sci. Adv. 2, e1600304 (2016).

[67] Y. Ohuchi, J. Matsuno, N. Ogawa, Y. Kozuka, M. Uchida, Y. Tokura, and M. Kawasaki, Electric-field control of anomalous and topological Hall effects in oxide bilayer thin films, Nat. Commun. 9, 213 (2018).

[68] Z. Li, S. Shen, Z. Tian, K. Hwangbo, M. Wang, Y. Wang, F. M. Bartram, L. He, Y. Lyu, Y. Dong et al., Reversible manipulation of the magnetic state in $\mathrm{SrRuO}_{3}$ through electric-field controlled proton evolution, Nat. Commun. 11, 184 (2020).

[69] D. Kan, K. Kobayashi, and Y. Shimakawa, Electric field induced modulation of transverse resistivity anomalies in ultrathin $\mathrm{SrRuO}_{3}$ epitaxial films, Phys. Rev. B 101, 144405 (2020).

[70] D. J. Groenendijk, C. Autieri, T. C. van Thiel, W. Brzezicki, J. R. Hortensius, D. Afanasiev, N. Gauquelin, P. Barone, K. H. W. van den Bos, S. van Aert et al., Berry phase engineering at oxide interfaces, Phys. Rev. Research 2, 023404 (2020).

[71] Y. Sugimoto, M. S. Anwar, S. R. Lee, Y. J. Shin, S. Yonezawa, T. W. Noh, and Y. Maeno, Ferromagnetic properties of $\mathrm{SrRuO}_{3}$ thin films deposited on the spin-triplet superconductor $\mathrm{Sr}_{2} \mathrm{RuO}_{4}$, Phys. Proc. 75, 413 (2015). 
[72] R. I. Salikhov, I. A. Garifullin, N. N. Garifyanov, L. R. Tagirov, K. Theis-Br'ohl, K. Westerholt, and H. Zabel, Inverse Proximity Effect in Superconductor-Ferromagnet Bilayer Structures, Phys. Rev. Lett. 102, 087003 (2009).

[73] J. Xia, V. Shelukhin, M. Karpovski, A. Kapitulnik, and A. Palevski, Experimental Observation of the Spin Screening Effect in Superconductor/Ferromagnet Thin Film Heterostructures, Phys. Rev. Lett. 102, 087004 (2009).

[74] Yu. N. Khaydukov, B. Nagy, J.-H. Kim, T. Keller, A. R'uhm, Yu. V. Nikitenko, K. N. Zhernenkov, J. Stahn, L. F. Kiss, A. Csik, L. Bottyán, and V. L. Aksenov, On the feasibility to study inverse proximity effect in a single $\mathrm{S} / \mathrm{F}$ bilayer by polarized neutron reflectometry, JETP Lett. 98, 107 (2013).

[75] B. Nagy, Yu. Khaydukov, D. Efremov, A. S. Vasenko, L. Mustafa, J.-H. Kim, T. Keller, K. Zhernenkov, A. Devishvili, R. Steitz, B. Keimer, and L. Bottyrán, On the explanation of the paramagnetic Meissner effect in superconductor/ferromagnet heterostructures, Europhys. Lett. 116, 17005 (2016).

[76] A. Di Bernardo et al., Intrinsic Paramagnetic Meissner Effect Due to S-Wave Odd-Frequency Superconductivity, Phys. Rev. X 5, 041021 (2015).

[77] M. G. Flokstra, N. Satchell, J. Kim, G. Burnell, P. J. Curran, S. J. Bending, J. F. K. Cooper, C. J. Kinane, S. Langridge, A. Isidori, N. Pugach, M. Eschrig, H. Luetkens, A. Suter, T. Prokscha, and S. L. Lee, Remotely induced magnetism in a normal metal using a superconducting spin-valve, Nat. Phys. 12, 57 (2016).

[78] M. G. Flokstra, R. Stewart, N. Satchell, G. Burnell, H. Luetkens, T. Prokscha, A. Suter, E. Morenzoni, S. Langridge, and S. L. Lee, Observation of Anomalous Meissner Screening in $\mathrm{Cu} / \mathrm{Nb}$ and $\mathrm{Cu} / \mathrm{Nb} / \mathrm{Co}$ Thin Films, Phys. Rev. Lett. 120, 247001 (2018).

[79] C. F. Majrkzak, Neutron scattering studies of magnetic thin films and multilayers, Physica B 221, 342 (1996).

[80] C. F. Majkrzak, K. V. O'Donovan, and N. F. Berk, Neutron Scattering from Magnetic Materials, edited by T. Chatterji (Elsevier, Amsterdam, 2006), Vol. 9.

[81] Y. Khaydukov (private communication).

[82] J. A. Krieger et al., Proximity-Induced Odd-Frequency Superconductivity in a Topological Insulator, Phys. Rev. Lett. 125, 026802 (2020).

[83] Zh. Devizorova, S. V. Mironov, A. S. Mel'nikov, and A. Buzdin, Electromagnetic proximity effect controlled by spin-triplet correlations in superconducting spin-valve structures, Phys. Rev. B 99, 104519 (2019).
[84] A. F. Volkov, F. S. Bergeret, and K. B. Efetov, Spin polarization and orbital effects in superconductor-ferromagnet structures, Phys. Rev. B 99, 144506 (2019).

[85] P. Hohenberg and W. Kohn, Inhomogeneous electron gas, Phys. Rev. 136, B864 (1964).

[86] W. Kohn and L. J. Sham, Self-consistent equations including exchange and correlation effects, Phys. Rev. 140, A1133 (1964).

[87] G. Kresse and D. Joubert, From ultrasoft pseudopotentials to the projector augmented-wave method, Phys. Rev. B 59, 1758 (1999).

[88] J. P. Perdew and A. Zunger, Self-interaction correction to density-functional approximations for many-electron systems, Phys. Rev. B 23, 5048 (1981)

[89] D. M. Ceperley and B. J. Alder, Ground State of the Electron Gas by a Stochastic Method, Phys. Rev. Lett. 45, 566 (1980).

[90] C. Etz, I. V. Maznichenko, D. Böttcher, J. Henk, A. N. Yaresko, W. Hergert, I. I. Mazin, I. Mertig, and A. Ernst, Indications of weak electronic correlations in $\mathrm{SrRuO}_{3}$ from first-principles calculations, Phys. Rev. B 86, 064441 (2012).

[91] P. E. Blöchl, Projector augmented-wave method, Phys. Rev. B 50, 17953 (1994).

[92] H. J. Monkhorst and J. D. Pack, Special points for Brillouinzone integrations, Phys. Rev. B 13, 5188 (1976).

[93] P. E. Blöchl, O. Jepsen, and O. K. Andersen, Improved tetrahedron method for Brillouin-zone integrations, Phys. Rev. B 49, 16223 (1994).

[94] V. I. Anisimov, J. Zaanen, and O. K. Andersen, Band theory and Mott insulators: Hubbard U instead of Stoner I, Phys. Rev. B 44, 943 (1991).

[95] V. I. Anisimov, I. V. Solovyev, M. A. Korotin, M. T. Czyzyk, and G. A. Sawatzky, Density-functional theory and NiO photoemission spectra, Phys. Rev. B 48, 16929 (1993).

[96] A. I. Liechtenstein, V. I. Anisimov, and J. Zaanen, Densityfunctional theory and strong interactions: Orbital ordering in Mott-Hubbard insulators, Phys. Rev. B 52, R5467(R) (1995).

[97] C. Autieri, M. Cuoco, and C. Noce, Collective properties of eutectic ruthenates: Role of nanometric inclusions, Phys. Rev. B 85, 075126 (2012).

[98] C. Autieri, M. Cuoco, and C. Noce, Structural and electronic properties of $\mathrm{Sr}_{2} \mathrm{RuO}_{4} / \mathrm{Sr}_{3} \mathrm{Ru}_{2} \mathrm{O}_{7}$, Phys. Rev. B 89, 075102 (2014). 